Received: 9 May 2017

Accepted: 25 August 2017

Published online: 11 September 2017

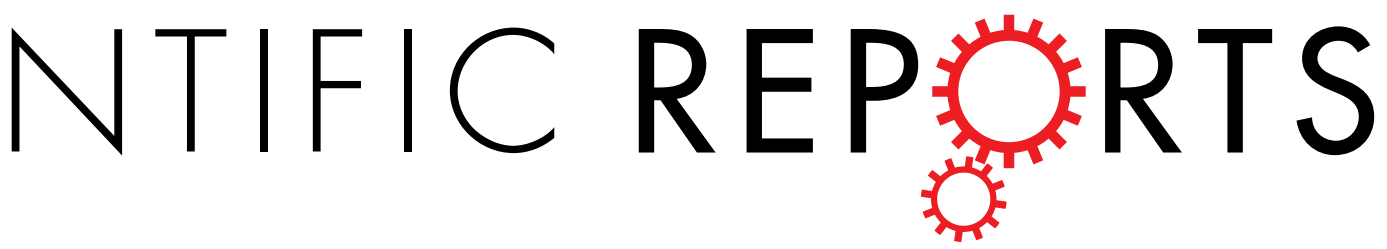

\title{
OPEN Agerarin, identified from Ageratum houstonianum, stimulates circadian CLOCK-mediated aquaporin-3 gene expression in $\mathrm{HaCaT}$ keratinocytes
}

Soon Young Shin ${ }^{1,2}$, Da Hyun Lee ${ }^{1}$, Ha-Na Gil2 ${ }^{2}$, Beom Soo Kim³ ${ }^{3}$, Jeong-Sook Choe ${ }^{4}$, Jung-Bong $\mathrm{Kim}^{4}$, Young Han Lee ${ }^{1,2}$ \& Yoongho Lim $^{3}$

The juice of Ageratum houstonianum is used in folk medicine as an external wound healing aid for skin injuries. However, the active component of $A$. houstonianum and its mode of action in skin wound healing has not been investigated. This study was conducted to investigate the effect of A. houstonianum ethanolnolic extract (AHE) on the expression of aquaporin-3 (AQP3), an integral membrane protein for water and glycerol transport in keratinocytes, and to identify the structure of the $A$. houstonianum bioactive compound. Here, we show that AHE increased AQP3 gene expression at the transcriptional level through the p38 MAPK pathway in $\mathrm{HaCaT}$ cells. Furthermore, AHE ameliorated suppression of $A Q P 3$ expression caused by ultraviolet B (UVB) irradiation. Agerarin (6,7-dimethoxy-2,2dimethyl-2H-chromene) was identified as the bioactive compound responsible for the up-regulation of $A Q P 3$ expression by enhancing the expression of the transcription factor circadian locomotor output cycles kaput (CLOCK). In conclusion, agerarin is a bioactive compound in AHE responsible for CLOCKmediated AQP3 expression in keratinocytes.

Water makes up about $60 \%$ of human body weight and plays an important role in maintaining tissue homeostasis in all biological physiologies. The skin contains about $30 \%$ of total body water and serves a waterproofing function for the body ${ }^{1}$. The skin is the soft, outer covering tissue and plays a key role in protecting the body against hazardous environmental threats, such as pathogens, external chemical insults, heat, and water loss. It is a highly organized multilayered epithelium, divided into two compartments: the epidermis and dermis. The epidermis is subdivided into several layers, including the stratum corneum, stratum granulosum, stratum spinosum, and stratum basale. The stratum corneum is located at the outermost part of the body and thereby functions as the primary permeability barrier, preventing water loss from the skin surface ${ }^{2}$.

Multiple complex factors are involved in the skin wound healing process. It is generally accepted that maintenance of skin hydration is important for the healing of various skin injuries ${ }^{3}$. Failure of skin water homeostasis causes delayed wound healing, hypertrophic scarring ${ }^{4}$, and development of various skin diseases, such as psoriasis ${ }^{5}$ and atopic dermatitis $s^{6,7}$. Furthermore, a decrease in the hydration level of the epidermis directly up-regulates the expression of various pro-inflammatory cytokines, including interleukin (IL)-1 $\beta$, IL-8, tumor necrosis factor- $\alpha$, and matrix metalloproteinase 9, demonstrating that epithelial hydration status plays a critical role in optimizing wound healing ${ }^{8}$.

Aquaporins (AQPs) are integral pore proteins that transport water molecules across the membrane ${ }^{9}$. In humans, at least 13 AQPs (AQP0-AQP12) have been identified to date. There are two types of AQPs: water-selective transporters such as AQP0, AQP2, AQP4, and AQP5; and water and glycerol transporters such as AQP3, AQP7, and AQP9 9 . Of these, AQP3 is highly expressed in the plasma membranes of keratinocytes and functions as both a water and glycerol transporter (aquaglyceroporin) in the basal layer of the skin epidermis ${ }^{2,10}$.

${ }^{1}$ Department of Biological Sciences, Sanghuh College of Life Sciences, Konkuk University, Seoul, 05029, Republic of Korea. ${ }^{2}$ Cancer and Metabolism Institute, Konkuk University, Seoul, 05029, Republic of Korea. ${ }^{3}$ Division of Bioscience and Biotechnology, BMIC, Konkuk University, Seoul, 05029, Republic of Korea. ${ }^{4}$ Department of Agrofood Resources, National Academy of Agricultural Sciences, Rural Development Administration, Jeonju, 55365, Republic of Korea. Correspondence and requests for materials should be addressed to Y.H.L. (email: yhlee58@konkuk.ac.kr) or Y.L. (email:yoongho@konkuk.ac.kr) 
AQP3-deficient mice have relatively dry skin and reduced skin elasticity as compared to wild-type mice ${ }^{11}$. A selective decrease in glycerol levels in AQP3-deficient mice may account for impaired skin hydration, elasticity, and barrier function ${ }^{12}$. In addition, AQP3 is believed to be important in wound healing by enhancing epidermal cell migration and proliferation ${ }^{3}$.

Ageratum houstonianum, a member of the Asteraceae family, is commonly known as flossflower, bluemink, blueweed, pussy foot, or Mexican paintbrush (Supplemental Fig. S1). It is a cool-season annual plant growing to a height of $0.3-1 \mathrm{~m}$, with ovate to triangular leaves and blue (sometimes white, pink, or purple) flower heads (In: PlantNET, The NSW Plant Information Network System, Royal Botanic Gardens and Domain Trust, Sydney; http://plantnet.rbgsyd.nsw.gov.au org). The plant is apparently native to Central America and southeastern Mexico and has been cultivated and naturalized in North America, Africa, Asia, Europe and Oceania (In: Invasive Species Compendium, Wallingford, UK; http://www.cabi.org/isc). Various bioactive components have been isolated from the plant, including flavones, pyrrolizidine alkaloids, steroids, benzofuran, and precocenes ${ }^{13-16}$. It has been reported that $A$. houstonianum has broad biological activities, including antifungal, antibacterial, and antimicrobial activities ${ }^{16-19}$. It has been used for treating pain and infections, especially for healing of external wounds and skin diseases ${ }^{15,20}$. However, the active component of $A$. houstonianum and its mode of action for curing skin wounds have not been investigated.

In this study, we aimed to investigate the effects of A. houstonianum ethanolic extract (AHE) on the expression of aquaporin-3 (AQP3). Our results demonstrate that agerarin can be used as a potential adjunct to enhance the treatment of various skin diseases by providing adequate hydration in the epidermis.

\begin{abstract}
Results
AHE up-regulates AQP3 expression in HaCaT keratinocytes. To investigate whether AHE alters the expression of the AQP3 gene, we first measured AQP3 mRNA levels in AHE-treated HaCaT human immortalized keratinocytes. HaCaT cells were treated with different concentrations $(0,5,10$, and $20 \mu \mathrm{g} / \mathrm{mL})$ of AHE, and levels of $A Q P 3$ mRNA were measured by reverse transcription-polymerase chain reaction (RT-PCR) analysis. As shown in Fig. 1a, AQP3 mRNA expression was substantially elevated by AHE treatment in a dose-dependent manner. Quantitative real-time PCR (qRT-PCR) analysis was conducted to precisely quantify the dose effect of AHE on $A Q P 3$ mRNA expression. AQP3 mRNA levels were significantly increased $3.7 \pm 0.57$-fold $(P=0.0013)$ and $6.6 \pm 0.96$-fold $(P<0.0001)$ after treatment of $A H E$ at 10 and $20 \mu \mathrm{g} / \mathrm{mL}$, respectively, compared with basal levels (Fig. 1b). To determine whether AHE-induced $A Q P 3$ up-regulation occurred at the transcriptional level, an $A Q P 3$ promoter reporter, pAQP3-Luc $(-1090 /+16)$, was generated and transfected into HaCaT keratinocytes. As shown in Fig. 1c, the luciferase reporter activity under control of the $A Q P 3$ gene promoter was significantly $(P<0.001)$ elevated when the cells were treated with AHE $(\geq 10 \mu \mathrm{g} / \mathrm{mL})$. A time-course experiment revealed that increases in $A Q P 3 \mathrm{mRNA}$ were detectable as early as $6 \mathrm{~h}$ after AHE treatment, and levels gradually increased in a time-dependent fashion (Fig. 1d). qRT-PCR showed that $A Q P 3 \mathrm{mRNA}$ levels were significantly increased $5.5 \pm 0.75$-fold $(P=0.0268)$ and $7.0 \pm 0.86$-fold $(P=0.0201)$ compared with basal levels after 12 and $24 \mathrm{~h}$, respectively (Fig. 1e). To further evaluate whether AHE induced the accumulation of AQP3 proteins, immunoblot analysis was performed to detect proteins in the lysates of AHE-treated HaCaT keratinocytes. In accordance with RT-PCR analysis, treatment with AHE led to a time-dependent elevation in the amount of AQP3 protein (Fig. 1f). We confirmed AHE-induced expression of AQP3 proteins by flow cytometry. AHE dose-dependently elevated the population of AQP3-positive cells (Fig. 1g), suggesting that AHE-induced AQP3 proteins are located on the cell surface membrane. Thus, the AQP3 gene is up-regulated at the transcriptional level by AHE treatment in $\mathrm{HaCaT}$ keratinocytes.
\end{abstract}

Inhibition of p38 MAPK abrogates AHE-induced AQP3 mRNA expression. Previous studies have demonstrated that $A Q P 3$ expression is regulated by the mitogen-activated protein kinase (MAPK) pathway ${ }^{21}$. To gain insight into the regulatory mechanism controlling $A Q P 3$ up-regulation in HaCaT cells, we examined the effect of AHE on the activation of three major MAPKs: ERK1/2, JNK1/2, and p38 MAPK. Serum-starved $\mathrm{HaCaT}$ cells were treated with $20 \mu \mathrm{g} / \mathrm{mL}$ AHE for various lengths of time, and MAPK activation was assessed using phospho-specific antibodies. We found that levels of phosphorylated ERK1/2 gradually decreased, whereas increased levels of phosphorylated JNK1/2 and p38 MAPK were detected within 10 min of AHE treatment (Fig. 2a). To assess the potential involvement of MAPK members in mediating AHE-induced AQP3 upregulation, specific chemical inhibitors were utilized. Pretreatment of HaCaT cells with the p38 MAPK inhibitor SB203580 significantly $(P<0.0001)$ abrogated AHE-induced accumulation of $A Q P 3 \mathrm{mRNA}$, whereas the MAPK kinase $1 / 2$ (MEK1/2) inhibitor U0126 and the JNK inhibitor SP600125 had little effect, as revealed by RT-PCR (Fig. 2b) and qRT-PCR (Fig. 2c). At present, although it is difficult to define the roles of the different MAPK pathways, it seems likely that p38 MAPK, at least in part, mediates AHE-induced AQP3 mRNA expression.

UVB irradiation reduces $A Q P 3$ expression in $\mathrm{HaCaT}$ keratinocytes. It has been reported that ultraviolet irradiation down-regulates $A Q P 3$ expression $^{22}$. UVB irradiation over $20 \mathrm{~mJ} / \mathrm{cm}^{2}$ reduced $A Q P 3 \mathrm{mRNA}$ expression in HaCaT cells, as revealed by RT-PCR (Fig. 3a). qRT-PCR showed that the relative expression of $A Q P 3$ mRNA was significantly decreased $0.4 \pm 0.1$-fold and $0.18 \pm 0.076$-fold (all $P<0.0001$ ) compared to levels in untreated controls at doses of 30 and $40 \mathrm{~mJ} / \mathrm{cm}^{2}$, respectively (Fig. 3b). Time-course analysis showed that UVB-induced down-regulation of $A Q P 3 \mathrm{mRNA}$ expression began to be detected after $6 \mathrm{~h}$ of UVB irradiation $\left(30 \mathrm{~mJ} / \mathrm{cm}^{2}\right)$, as revealed by RT-PCR (Fig. 3c). Similar results were obtained by qRT-PCR (Fig. 3d). These data demonstrate that UVB irradiation at a dose of $30 \mathrm{~mJ} / \mathrm{cm}^{2}$ for longer than $6 \mathrm{~h}$ down-regulates $A Q P 3$ expression in HaCaT keratinocytes. 
(a)

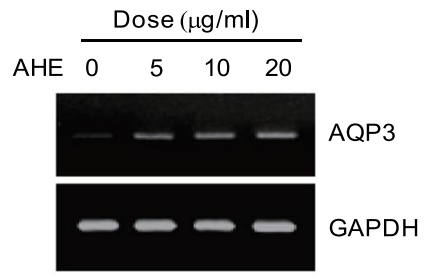

(d)

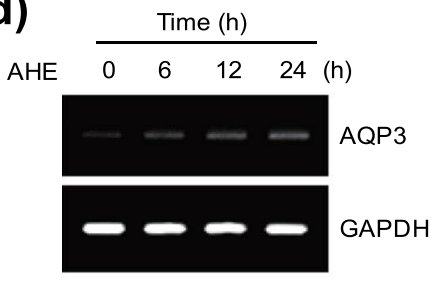

(g)

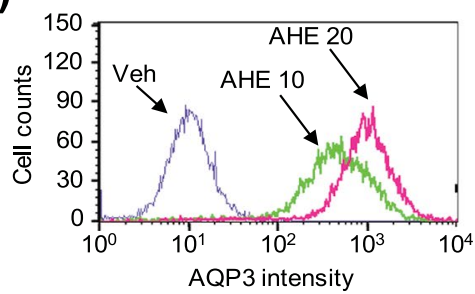

(b)

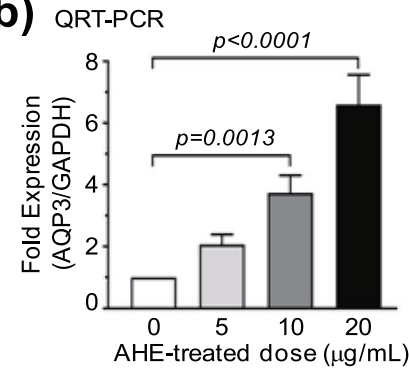

(e)

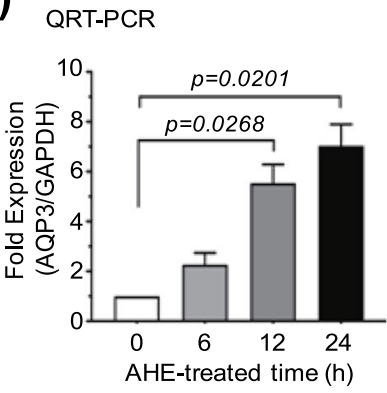

(c)

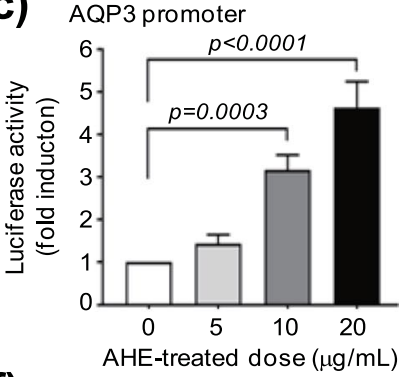

(f)

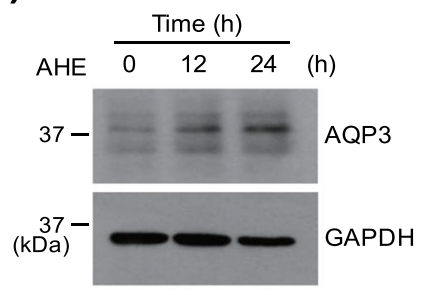

Figure 1. Effect of AHE on AQP3 expression. (a) HaCaT cells were treated with different concentrations of AHE for $24 \mathrm{~h}$. AQP3 mRNA expression was analyzed by RT-PCR. GAPDH expression was used as an internal control. (b) HaCaT cells were treated with as in (a). AQP3 mRNA levels were quantitated by qRT-PCR. GAPDH mRNA level was used for normalization. (c) AQP3 promoter assay. pAQP3-Luc $(-1090 /+16)$ reporter was transfected into HaCaT cells. After $48 \mathrm{~h}$, cells were treated with different concentrations of AHE. After 8-12 h, cells were collected and luciferase activities were measured. (d) HaCaT cells were treated with $20 \mu \mathrm{g} / \mathrm{mL}$ AHE for various time periods. AQP3 mRNA expression was analyzed by RT-PCR. GAPDH expression was used as an internal control. (e) HaCaT cells were treated as in (d). AQP3 mRNA levels were measured by qRT-PCR. The relative fold changes were normalized to the expression of GAPDH mRNA. (f) HaCaT cells were treated with $20 \mu \mathrm{g} / \mathrm{mL}$ AHE for various time periods. AQP3 protein levels were analyzed by immunoblotting. GAPDH expression was used as an internal control. (g) Flow cytometry. HaCaT cells were treated with AHE (10 and $20 \mu \mathrm{g} / \mathrm{mL}$ ) and the percentage of AQP3 positive cells were measured by flow cytometry. Full-length gels (a and d) and blots (f) are presented in Supplemental Fig. S12.

AHE overcomes UVB-induced suppression of $A Q P 3$ expression. We next attempted to determine whether AHE has the ability to ameliorate ultraviolet B (UVB)-induced suppression of $A Q P 3$ expression. HaCaT cells were treated with AHE for $30 \mathrm{~min}$, followed by irradiation with UVB $\left(30 \mathrm{~mJ} / \mathrm{cm}^{2}\right)$. As shown in Fig. $4 \mathrm{a}$, UVB-induced suppression of AQP3 mRNA expression was gradually ameliorated by AHE treatment. qRT-PCR showed that levels of $A Q P 3$ mRNA significantly $(P<0.0001)$ increased about 2 -fold compared with basal levels following pretreatment with $>10 \mu \mathrm{g} / \mathrm{mL}$ AHE (Fig. 4b). Moreover, UVB-induced suppression of both AQP3 promoter activity (Fig. 4c) and AQP3 protein accumulation (Fig. 4d) was inhibited by AHE treatment. We confirmed the effect of AHE on the accumulation of AQP3 protein by immunofluorescence microscopy. HaCaT cells cultured on a coverglass were pretreated with AHE before UVB irradiation. As shown in Fig. 4e, the reduction in AQP3 protein staining following UVB irradiation was inhibited by AHE treatment. These results suggest that $\mathrm{AHE}$ is able to overcome UVB-induced suppression of $A Q P 3$ expression.

Identification of agerarin as an active compound of AHE. To identify the active component responsible for AQP3 up-regulation, AHE was further fractionated using $\mathrm{n}$-hexane, chloroform, and water (Supplemental Fig. S2a). We observed that $\mathrm{n}$-hexane fraction (AHE-Hx) significantly $(P<0.0001)$ inhibited UVB-induced suppression of $A Q P 3$ expression (Supplemental Fig. S2b) and was able to induce AQP3 mRNA expression (Supplemental Fig. S2c), suggesting that AHE-Hx contains active components responsible for the induction of $A Q P 3$. AHE-Hx fraction was further separated by a prep-high-performance liquid chromatography (prep-HPLC) with a Luna C18 column. As shown in Fig. 5a, a major peak was observed at $8.6 \mathrm{~min}$ in the chromatogram. The peak (named AG-H1) was a single compound as revealed by analysis with a photodiode array detector (Fig. 5b). To elucidate the chemical structure, NMR spectroscopy was carried out using a Bruker AVANCE 400 spectrometer system. Twelve peaks were observed in the ${ }^{13} \mathrm{C}$ nuclear magnetic resonance (NMR) spectrum (Supplemental Fig. S3), and seven peaks were observed in the ${ }^{1} \mathrm{H}$ NMR spectrum (Supplemental Fig. S4). Distortionless 
(a)

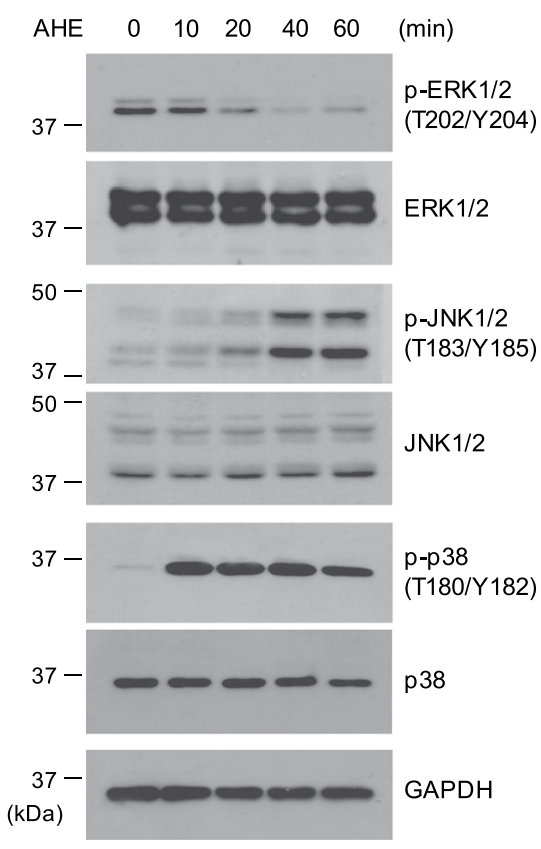

(b)

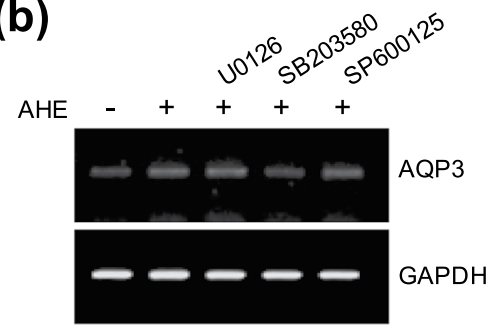

(c)

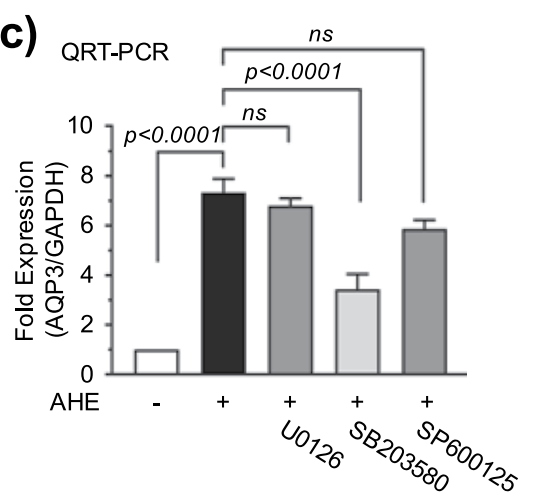

Figure 2. Role of MAPK signaling in AHE-induced AQP3 expression. (a) HaCaT cells were treated with $20 \mu \mathrm{g} /$ $\mathrm{mL}$ AHE for various lengths of time. Phosphorylation status of MAPKs were analyzed by immunoblotting. GAPDH antibody was used as an internal control to show equal protein loading. (b) HaCaT cells were either untreated or pretreated with U0126 $(10 \mu \mathrm{M})$, SB203580 $(20 \mu \mathrm{M})$, or SP600125 $(25 \mu \mathrm{M})$ before addition of $20 \mu \mathrm{g} / \mathrm{mL}$ AHE for $24 \mathrm{~h}$. AQP3 mRNA expression was analyzed by RT-PCR. GAPDH expression was used as an internal control. (c) HaCaT cells were treated as in (b). AQP3 mRNA levels were measured by qRT-PCR. GAPDH mRNA level was used for normalization. ns, not significant. Full-length blots (a) and gels (b) are presented in Supplemental Fig. S13.

(a)

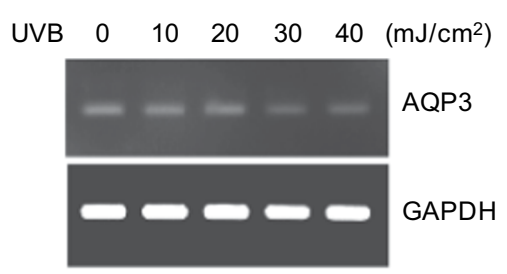

(b)

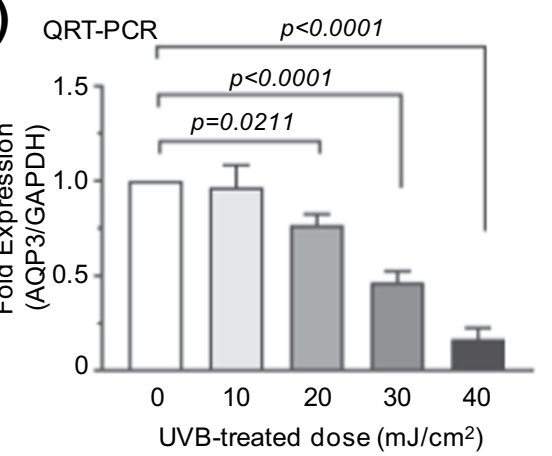

(c)

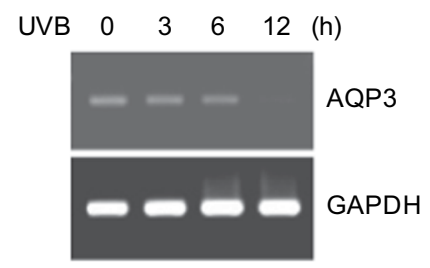

(d)

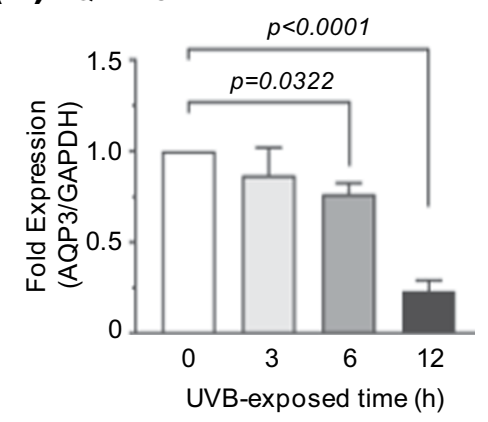

Figure 3. Effect of UVB on the suppression of AQP3 expression. (a) RT-PCR. HaCaT cells were irradiated with different doses of UVB for $24 \mathrm{~h}$. AQP3 mRNA expression was analyzed by RT-PCR. GAPDH expression was used as an internal control. (b) HaCaT cells were treated with as in (a). AQP3 mRNA levels were measured by qRT-PCR. GAPDH mRNA level was used for normalization. (c) HaCaT cells were irradiated with UVB (30 mJ/ $\mathrm{cm}^{2}$ ) for various time periods. AQP3 mRNA expression was analyzed by RT-PCR. GAPDH expression was used as an internal control. (d) HaCaT cells were irradiated with UVB as in (c). AQP3 mRNA levels were quantitated by qRT-PCR. The mRNA expression were normalized to the GAPDH mRNA. Full-length gels (a and c) are presented in Supplemental Fig. S14. 
(a)
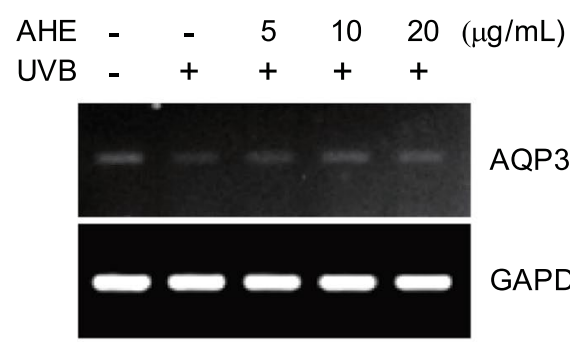

AQP3

GAPDH

(b)

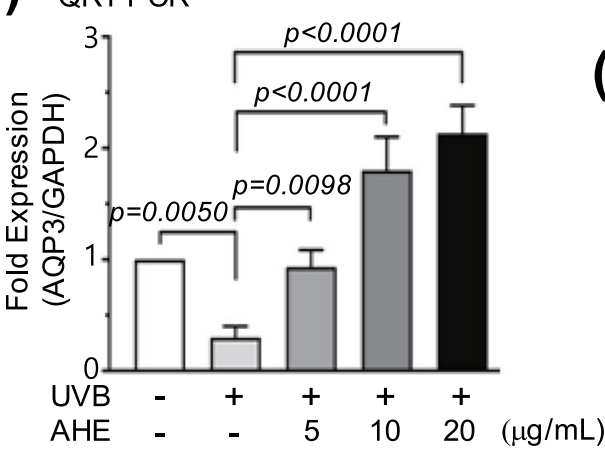

(e)
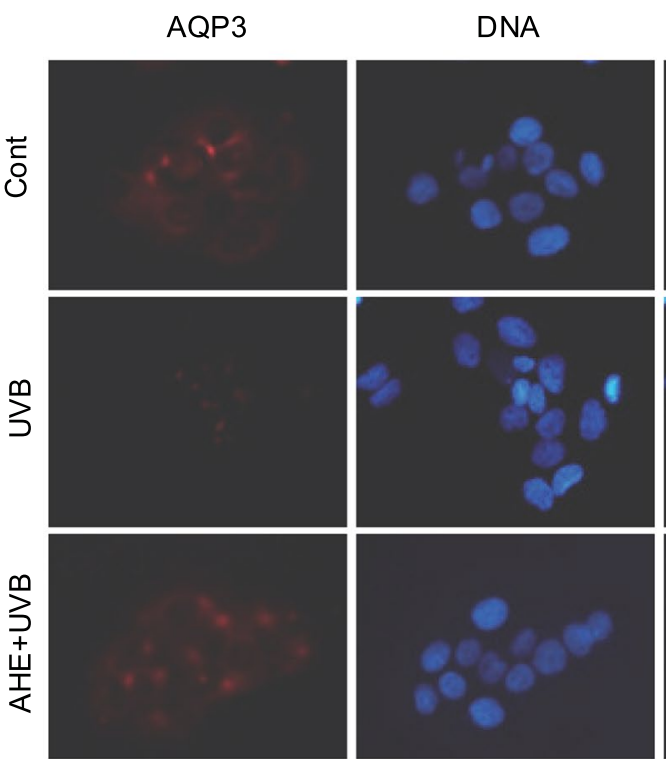

(c) $\mathrm{AQP} 3$ promoter

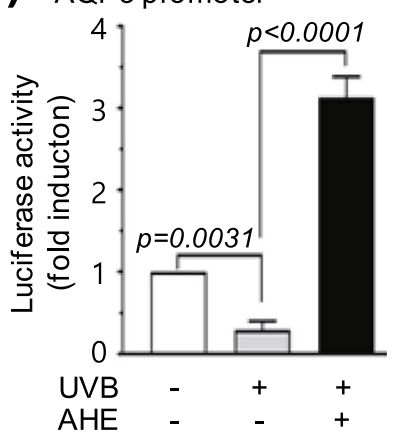

(d)

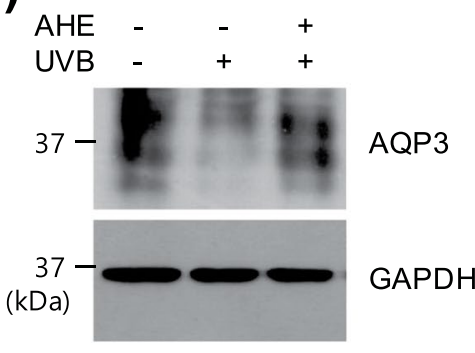

Figure 4. Effect of AHE on UVB-induced suppression of AQP3 expression. (a) HaCaT cells were irradiated with UVB $\left(30 \mathrm{~mJ} / \mathrm{cm}^{2}\right)$ in the absence or presence of different concentrations of AHE. After $24 \mathrm{~h}, A Q P 3 \mathrm{mRNA}$ expression was analyzed by RT-PCR. GAPDH expression was used as an internal control. (b) HaCaT cells were treated as in (a) and qRT-PCR was performed. GAPDH mRNA level was used for normalization. (c) HaCaT cells were transfected with $0.2 \mu \mathrm{g}$ of promoter reporter pAQP3-Luc $(-1090 /+16)$, followed by irradiation with $\mathrm{UVB}\left(30 \mathrm{~mJ} / \mathrm{cm}^{2}\right)$ in the absence or presence of $20 \mu \mathrm{g} / \mathrm{mL}$ AHE at $24 \mathrm{~h}$ post-transfection. After $8-12 \mathrm{~h}$, luciferase activities were measured. (d) HaCaT cells were irradiated with UVB $\left(30 \mathrm{~mJ} / \mathrm{cm}^{2}\right)$ in the absence or presence of AHE $(20$ and $10 \mu \mathrm{g} / \mathrm{mL})$. After $24 \mathrm{~h}$, AQP3 protein level was detected by immunoblotting. GAPDH was used as an internal control. (e) HaCaT cells cultured on a coverglass were treated as in (d). AQP3 antibody was incubated for $2 \mathrm{~h}$, followed by AlexaFluor 555-conjugated (red signal) secondary antibody for additional $30 \mathrm{~min}$. Nuclear DNA was stained with $0.1 \mu \mathrm{g} / \mathrm{mL}$ Hoechst 33258 (blue signal) for $10 \mathrm{~min}$. Fluorescence-positive cells were examined under an EVOSf $1{ }^{\circledR}$ fluorescence microscope. Full-length gels (a) and blots (d) are presented in Supplemental Fig. S15. 
(a)

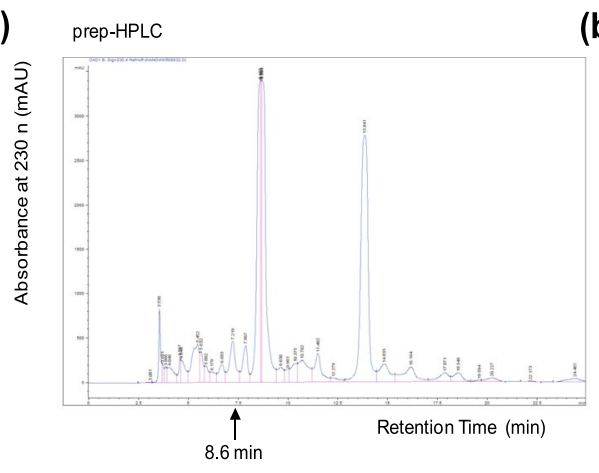

(c)

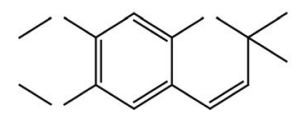

Agerarin (6,7-dimethoxy-2,2-dimethyl-2H-chromene ) (b)

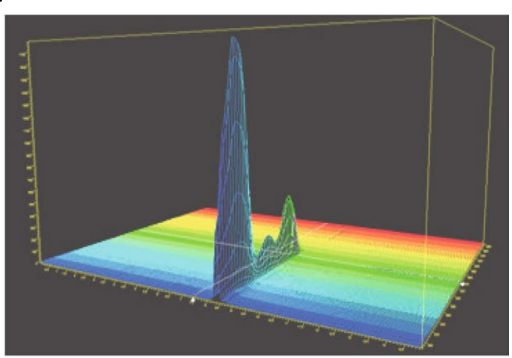

Figure 5. Identification of agerarin as an active component of AHE. (a) Chromatogram of agerarin at $230 \mathrm{~nm}$. (b) The peak detected at $8.6 \mathrm{~min}$ on the HPLC chromatogram with photodiode array detector at $230 \mathrm{~nm}$. (c) The structure of agerarin, 6,7-dimethoxy-2,2-dimethyl- $2 H$-chromene.

Enhancement by Polarization Transfer (DEPT) data showed that this compound consists of three quartet, four doublet, and five singlet carbons (Supplemental Fig. S5). Since two carbon peaks at 56.1 and 56.7 ppm were attached directly to the proton peaks at 3.80 and $3.78 \mathrm{ppm}$, respectively, in the heteronuclear multiple quantum coherence (HMQC) spectrum (Supplemental Fig. S6), they were assigned two methoxy groups. The proton peak at $1.38 \mathrm{ppm}$ attached directly to the carbon peak at $27.8 \mathrm{ppm}$ was assigned a methyl group, and they were determined to be two methyl groups based on the integration value of the proton peak. Nine carbon peaks and four proton peaks showed the pattern of the chromene scaffold ${ }^{23}$. Based on interpretation of the total correlated spectroscopy (TOCSY; Supplemental Fig. S7), correlated spectroscopy (COSY; Supplemental Fig. S8), heteronuclear multiple bonded connectivities (HMBC; Supplemental Fig. S9), and heteronuclear multiple quantum coherence (HMQC) spectra, this scaffold was identified as a $2 \mathrm{H}$-chromenone. The important correlations obtained from the interpretations of the COSY and HMBC spectra are provided as Supplemental Fig. S10. The complete assignments of the ${ }^{1} \mathrm{H}$ and ${ }^{13} \mathrm{C}$ NMR data are listed in Supplemental Table S1. As a result, the AG-H1 peak was identified as 6,7-dimethoxy-2,2-dimethyl-2H-chromene (PubChem CID: 12565; named agerarin; Fig. 5c). Agerarin is known as ageratochromene ${ }^{24}$ or precocene $\mathrm{II}^{25}$. To confirm this structure, High resolution mass spectrometry (HR/MS) was carried out with ultra-performance liquid chromatography-hybrid quadrupole/time-of-flight mass spectrometry (UPLC-Q-Tof-MS). The data were collected as M+H ions as shown in Supplemental Fig. S11. The calculated mass was 221.1178 , and the mass found by HR/MS was 221.5345. These data demonstrate that the agerarin structure determined by NMR spectroscopy agreed with that obtained by HR/MS.

Agerarin alone induces $A Q P 3$ mRNA expression. We next explored whether agerarin alone increases $A Q P 3$ expression. RT-PCR analysis shows that treatment with agerarin alone markedly increased AQP3 mRNA levels, comparable in magnitude to the increase with AHE treatment (Fig. 6a). qRT-PCR demonstrates that both AHE and agerarin increased $A Q P 3$ mRNA levels in a dose-dependent manner (Fig. 6b). At the highest dose $(20 \mu \mathrm{g} / \mathrm{mL})$, AHE and agerarin significantly increased AQP3 mRNA levels by 6.27 -fold and 7.17-fold, respectively, compared to vehicle control $(p<0.0001)$. There was a statistically significant difference between AHE and agerarin treated groups ( $P=0.0357$ by Sidak's mutiple comparisons test, $\mathrm{n}=3$ ). Furthermore, agerarin alone inhibited UVB-induced suppression of $A Q P 3$ mRNA expression, as revealed by RT-PCR (Fig. 6c). Similar results were obtained by qRT-PCR (Fig. 6d). These results demonstrate that agerarin isolated from AHE is responsible for the up-regulation of $A Q P 3$ expression.

Agerarin stimulates the circadian CLOCK gene expression. To investigate the molecular mechanism underlying AHE-induced $A Q P 3$ expression, we utilized agerarin. A previous report demonstrated that $A Q P 3$ expression in human keratinocytes is regulated by molecular clock genes, including Circadian Locomotor Output Cycles Kaput $(\mathrm{CLOCK})^{26}$. On the basis of this study, we considered the possibility that molecular clock genes play an important role in agerarin-induced transcriptional activation of the $A Q P 3$ gene. To test this possibility, we first examined whether CLOCK is required for the induction of $A Q P 3$ gene promoter activity. HaCaT cells were transfected with the AQP3 promoter reporter, pAQP3-Luc $(-1090 /+16)$, along with an expression plasmid for CLOCK. In accordance with a previous report ${ }^{26}$, transient transfection of the CLOCK gene resulted in stimulation of promoter reporter activity in a plasmid concentration-dependent manner (Fig. 7a). Moreover, a 5'-deletion construct $(-198 /+16)$ that lacks CLOCK binding sites (E-box) resulted in complete loss of CLOCK-induced promoter activation (Fig. 7b). These data suggest that CLOCK plays an important role in AQP3 transcriptional activation. A time-course experiment showed that levels of CLOCK mRNA increased within $3 \mathrm{~h}$, were maintained through $12 \mathrm{~h}$, and then decreased at $24 \mathrm{~h}$ after the addition of agerarin (Fig. 7c). qRT-PCR analysis revealed that 
(a)

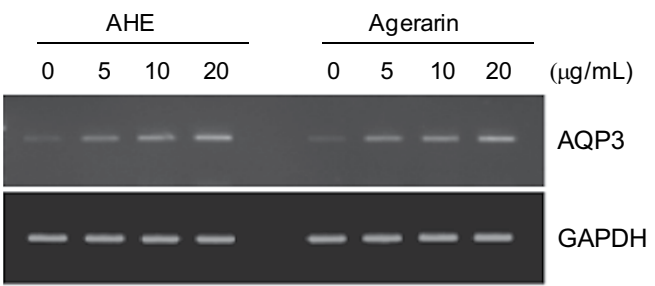

(b) QRT-PCR

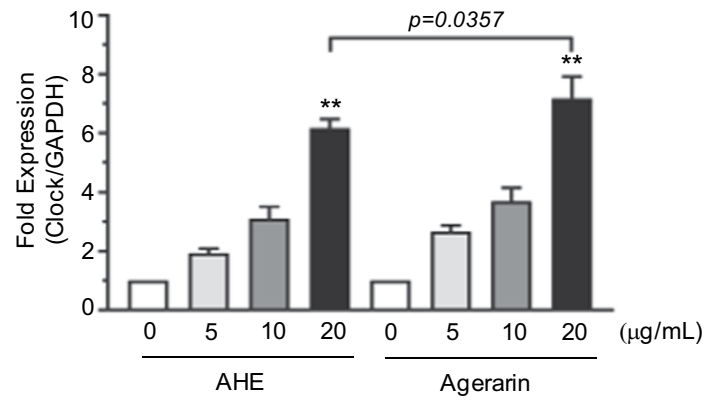

(c)

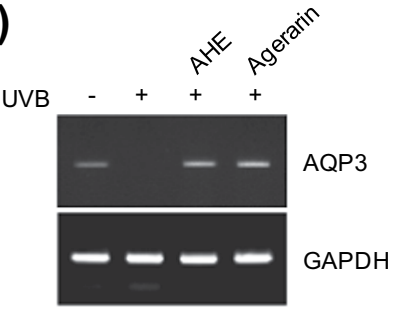

(d) $Q R T-P C R$

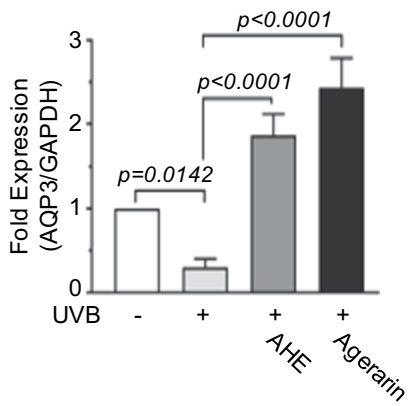

Figure 6. Effect of agerarin on the expression of $A Q P 3$. (a) HaCaT cells were treated with different concentrations of AHE or agerarin. After $24 \mathrm{~h}, A Q P 3$ mRNA expression was analyzed by RT-PCR. GAPDH expression was used as an internal control. (b) HaCaT cells were treated as in (a) and AQP3 mRNA levels were measured by qRT-PCR. The relative fold changes were normalized to the expression of GAPDH mRNA. $* * p<0.0001$ compared to vehicle control $(0 \mu \mathrm{g} / \mathrm{mL})$. (c) HaCaT cells were irradiated with UVB $\left(30 \mathrm{~mJ} / \mathrm{cm}^{2}\right)$ in the absence or presence of AHE or agerarin (each $20 \mu \mathrm{g} / \mathrm{mL}$ ). After $24 \mathrm{~h}, A Q P 3 \mathrm{mRNA}$ expression was analyzed by RT-PCR. GAPDH expression was used as an internal control. (d) HaCaT cells were treated as in (c) and qRTPCR was performed. GAPDH mRNA level was used for normalization. Full-length gels (a and $\mathbf{c}$ ) are presented in Supplemental Fig. S16.

agerarin-induced CLOCK mRNA levels were significantly increased $4.8 \pm 0.79$-fold $(P<0.0001), 7.0 \pm 0.57$-fold $(P<0.0001)$, and $3.3 \pm 0.45$-fold $(P=0.0021)$ compared with basal levels after 6,12 , and $24 \mathrm{~h}$, respectively (Fig. 7d). Immunoblot analysis showed similar results (Fig. 7e). To determine whether agerarin-induced CLOCK up-regulation occurred at the transcriptional level, a CLOCK promoter reporter, pClock-Luc $(-1000 /+47)$, was generated and transfected into HaCaT keratinocytes. As shown in Fig. $7 \mathrm{f}$, treatment with agerarin at 5, 10, and $20 \mu \mathrm{g} / \mathrm{mL}$ resulted in $1.9 \pm 0.46$-fold $(P>0.05), 3.8 \pm 0.7$-fold $(P=0.0003)$, and $6.7 \pm 0.50$-fold $(P<0.0001)$ increases, respectively, in luciferase reporter activity under the CLOCK gene promoter. These results demonstrate that $C L O C K$ expression is up-regulated by agerarin isolated from AHE and is involved in the transcriptional activation of the $A Q P 3$ gene in HaCaT keratinocytes.

Silencing of CLOCK abrogates agerarin-induced $A Q P 3$ upregulation. To corroborate the functional role of CLOCK in agerarin-induced $A Q P 3$ expression, we established $\mathrm{HaCaT}$ variant cell lines expressing scrambled control shRNA (shCont) or CLOCK shRNA (shClock), using a lentiviral expression system. Stable knockdown of CLOCK expression was verified by RT-PCR. We further confirmed the silencing of CLOCK expression after agerarin treatment for $12 \mathrm{~h}$, by RT-PCR (Fig. 8a) and qRT-PCR (Fig. 8b). We next addressed whether AQP3 expression is affected by the silencing of $C L O C K$ expression. HaCaT cells were treated with agerarin for $24 \mathrm{~h}$, and then AQP3 mRNA levels were measured by RT-PCR. Agerarin-induced AQP3 expression was markedly reduced in $\mathrm{HaCaT} / \mathrm{shClock}$ cells compared with that in HaCaT/shCont cells (Fig. 8c). qRT-PCR showed that agerarin-induced $A Q P 3$ mRNA expression was significantly $(P<0.0001)$ inhibited by the silencing of CLOCK (Fig. 8d). These results strongly implicate CLOCK as the transcription factor responsible for agerarin-induced $A Q P 3$ mRNA expression.

\section{Discussion}

The juice of $A$. houstonianum has been used in folk medicine for external wound healing of skin injuries in Korea, Mexico, India, and Nepal ${ }^{15,20}$. However, the mode of action of A. houstonianum in skin wound healing had not been investigated. Hydration status in the epidermis is important for maintenance of the skin barrier structure and the healing process following various skin injuries ${ }^{27}$. Aquaglyceroporin AQP3 plays an essential role in transporting both water and glycerol in the epidermal basal layer ${ }^{10}$. In this study, we evaluated the effect of $A$. houstonianum ethanolic extract (AHE) on the expression of $A Q P 3$ in human immortalized HaCaT keratinocytes. In addition, we identified agerarin as a bioactive component of AHE responsible for AQP3 up-regulation. To our knowledge, this is the first report showing the effects of AHE on AQP3 up-regulation in keratinocytes.

In the skin, AQP3 expressed on epidermal keratinocytes transports both water and glycerol from the dermis to the epidermis, thereby maintaining proper hydration levels in the epidermis. Glycerol is a substrate for the 
(a)

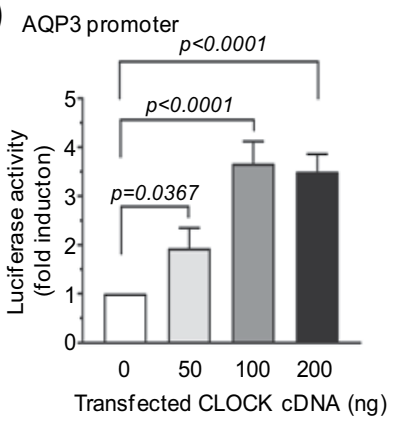

(c)

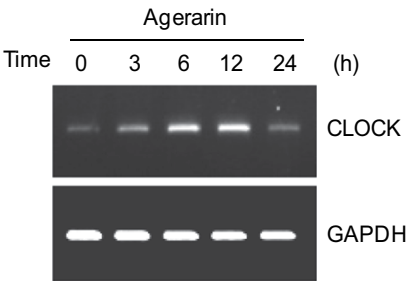

(d)

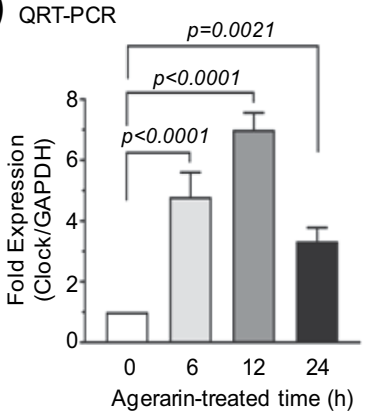

(b)

AQP3 promoter
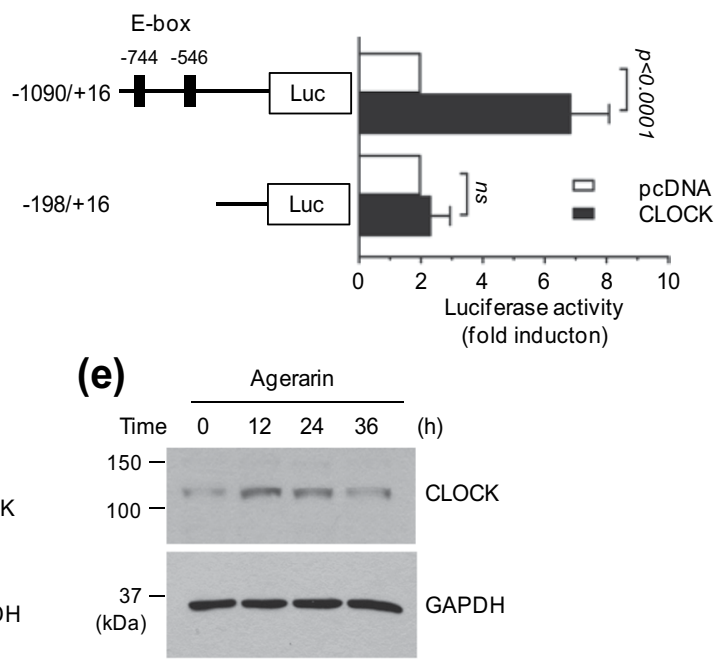

(f)

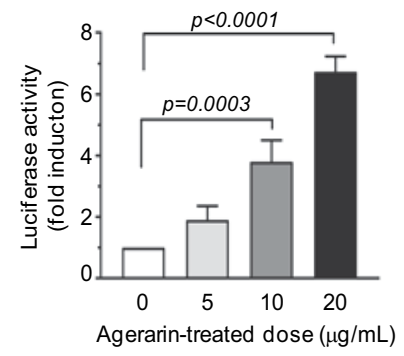

Figure 7. Effect of agerarin on the expression of CLOCK. (a) HaCaT cells were transfected with $0.2 \mu \mathrm{g}$ of promoter construct pAQP3-Luc $(-1090 /+16)$ along with different concentrations of expression plasmids for CLOCK, pcDNA3.1/Clock. After $24 \mathrm{~h}$, cells were collected and assayed for luciferase activity. (b) HaCaT cells were transfected with $0.2 \mu \mathrm{g}$ of a full-length $(-1090 /+16)$ or $5^{\prime}$-deletion construct $(-198 /+16)$ along with $0.1 \mu \mathrm{g}$ of empty vector (pcDNA3,1) or pcDNA3.1/Clock plasmids, and measured luciferase activity after $24 \mathrm{~h}$. (c) $\mathrm{HaCaT}$ cells were treated with agerarin $(20 \mu \mathrm{g} / \mathrm{mL})$ for various time periods. AQP3 mRNA expression was analyzed by RT-PCR. GAPDH expression was used as an internal control. (d) HaCaT cells were treated as in (b) and $A Q P 3$ mRNA levels were measured by qRT-PCR. GAPDH mRNA level was used for normalization. (e) $\mathrm{HaCaT}$ cells were treated with $20 \mu \mathrm{g} / \mathrm{mL}$ agerarin for various time periods. AQP3 protein level was detected by immunoblotting. GAPDH was used as an internal control. (f) CLOCK promoter assay. HaCaT cells were transfected with $0.2 \mu \mathrm{g}$ of promoter reporter pClock-Luc $(-1000 /-1)$. After $48 \mathrm{~h}$, HaCaT cells were treated with different concentrations of agerarin. After $12 \mathrm{~h}$, luciferase activities were measured. Full-length gels (c) and blots (e) are presented in Supplemental Fig. S17.

synthesis of triglycerides and various lipids in the living body. It has been demonstrated that AQP3-facilitated glycerol plays a critical role in the maintenance of skin hydration as well as in lipid metabolism and wound healing ${ }^{11}$. In this study, we found that AHE significantly elevated AQP3 mRNA expression in dose- and time-dependent manners. To determine whether AHE-induced $A Q P 3$ up-regulation occurred at the transcriptional level, we isolated the $5^{\prime}$-regulatory region of the human $A Q P 3$ gene located within $1.1 \mathrm{~kb}$ upstream of the transcriptional start site and subcloned this into a luciferase reporter vector, yielding pAQP3-Luc $(-1090 /+16)$. $A Q P 3$ gene promoter activity was significantly elevated by treatment with $>10 \mu \mathrm{g} / \mathrm{mL}$ AHE, suggesting that AHE stimulates expression of the $A Q P 3$ gene at the transcriptional level. We next identified the chemical structure of the active component of AHE, 6,7-dimethoxy-2,2-dimethyl-2H-chromene (named agerarin) as revealed by ${ }^{1} \mathrm{H}$ and ${ }^{13} \mathrm{C}$ NMR spectrometry and confirmed that agerarin is able to stimulate $A Q P 3$ gene transcription.

Skin hydration is controlled by circadian rhythms ${ }^{28}$. The circadian clock is a highly conserved molecular timing system that coordinates various metabolic and physiological processes within a period of $\sim 24 \mathrm{~h}$. In mammals, central circadian rhythms are regulated by a highly specialized master pacemaker located in the suprachiasmatic nucleus of the anterior hypothalamus, which receives and conveys environmental timing information to the local $\operatorname{organs}^{29}$. Peripheral tissue clocks also exist and are involved in the regulation of local metabolism and physiology ${ }^{30,31}$. Indeed, a recent study demonstrates that a natural flavone called Nobiletin acts on the circadian clock to enhance glucose metabolism ${ }^{32}$, suggesting that pharmacological modulation of circadian clock can regulate physiological responses. Both central and peripheral circadian clocks are regulated by circadian transcription factors, such as 
(a)

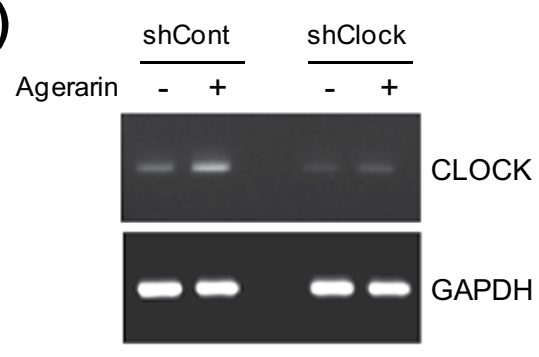

(b)

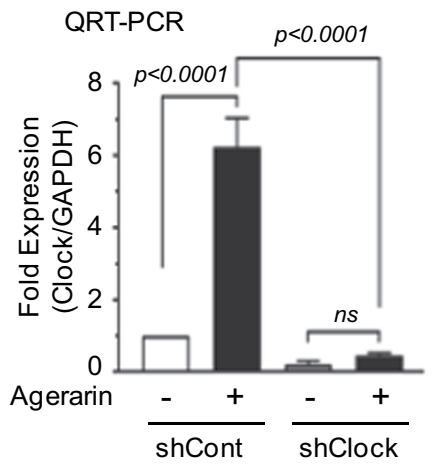

(c)

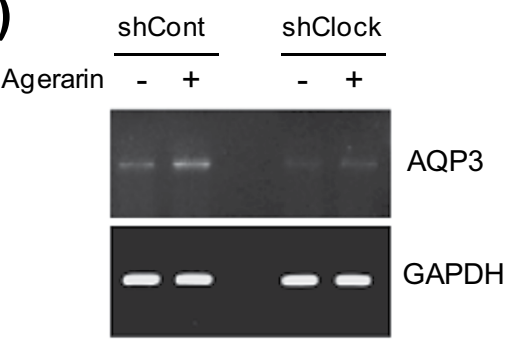

(d)

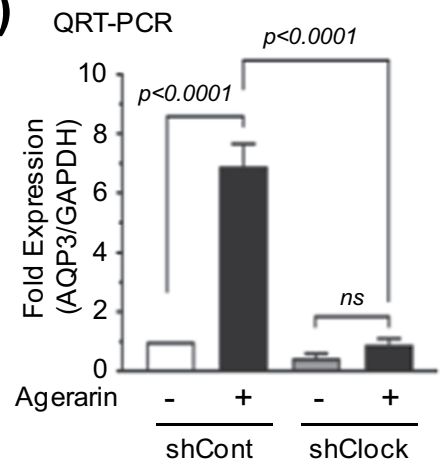

Figure 8. Effect of CLOCK silencing on agerarin-induced AQP3 expression. (a) HaCaT/shCont and $\mathrm{HaCaT} /$ shClock cells were treated with agerarin (each $20 \mu \mathrm{g} / \mathrm{mL}$ ) for $12 \mathrm{~h}$. CLOCK mRNA expression was analyzed by RT-PCR. GAPDH expression was used as an internal control. (b) HaCaT cells were treated as in (a) and CLOCK mRNA levels were measured by qRT-PCR. GAPDH mRNA level was used for normalization. (c) HaCaT/shCont and $\mathrm{HaCaT} / \mathrm{shClock}$ cells were treated with agerarin $(20 \mu \mathrm{g} / \mathrm{mL})$. After $24 \mathrm{~h}, A Q P 3 \mathrm{mRNA}$ expression was analyzed by RT-PCR. GAPDH expression was used as an internal control. (d) HaCaT cells were treated as in (c) and AQP3 mRNA levels were measured by qRT-PCR. GAPDH mRNA level was used for normalization. Fulllength gels (a and c) are presented in Supplemental Fig. S18.

CLOCK and BMAL1 (brain and muscle aryl hydrocarbon receptor nuclear translocator-like protein-1) ${ }^{29,33-35}$. Circadian clock genes are also expressed in the human skin $^{36}$ and are involved in temperature control and cholesterol metabolism ${ }^{37}$. AQP3 expression oscillates with a period length of about $24 \mathrm{~h}$, which is regulated by the CLOCK/BMAL1 circadian proteins in $\mathrm{HaCaT}$ keratinocytes ${ }^{26}$. In an effort to understand the molecular mechanism by which the $A Q P 3$ gene is regulated by agerarin, we tested whether the circadian gene CLOCK is involved in agerarin-induced $A Q P 3$ up-regulation. Our data showed that agerarin treatment caused the accumulation of CLOCK mRNA and protein levels within $6 \mathrm{~h}$ and $12 \mathrm{~h}$, respectively. Promoter reporter assay revealed that agerarin-induced up-regulation of $C L O C K$ is also regulated at the transcriptional level. As forced expression of CLOCK stimulated $A Q P 3$ promoter reporter activity, we hypothesized that agerarin-induced CLOCK targets the $A Q P 3$ gene in HaCaT keratinocytes. To corroborate the functional role of CLOCK in agerarin-induced AQP3 expression, we established $\mathrm{HaCaT}$ variant cell lines expressing scrambled control shRNA (HaCaT/shCont) or CLOCK shRNA (HaCaT/shClock), using a lentiviral expression system. Our data showed that stable silencing of CLOCK expression significantly attenuated agerarin-induced AQP3 expression, supporting the notion that CLOCK is directly involved in agerarin-induced $A Q P 3$ expression. Although further studies regarding the interactive mechanisms of agerarin-induced CLOCK transcription are required, our results demonstrate that agerarin enhances $A Q P 3$ gene expression through the activation of the circadian CLOCK protein.

MAPK signaling pathways, including ERK1/2, JNK1/2, and p38 MAPK, are well-known intracellular pathways that provide a mechanism for controlling the expression of tissue-specific target genes. Importantly, MAPK pathways are involved in the regulation of the circadian $\operatorname{clock}^{38}$. Our data showed that AHE rapidly induces the phosphorylation of JNK1/2 and p38 MAPK but not ERK1/2. We also observed that treatment with the p38 kinase inhibitor SB203580 but not with the JNK inhibitor SP600125 effectively inhibited AHE-induced AQP3 mRNA expression. These data suggest that the p38 MAPK signaling pathway may be involved in AHE-induced AQP3 expression in HaCaT keratinocytes. The role of p38 MAPK in regulating circadian rhythms in chicken ${ }^{39}$ and fruit fly ${ }^{40}$ has been demonstrated. The p38 MAPK pathway is also involved in TGF $\beta 1$-induced AQP3 up-regulation in human peritoneal mesothelial cells ${ }^{41}$. Thus, it is possible that agerarin-induced $A Q P 3$ up-regulation is mediated by 38 MAPK-dependent CLOCK expression. It would be interesting to address the role of p38 MAPK in the regulation of CLOCK-mediated $A Q P 3$ expression in future studies.

In conclusion, this study demonstrated that the ethanolic extract of A. houstonianum (AHE) has pharmacological activity, enhancing expression of the aquaglyceroporin AQP3 in HaCaT keratinocytes. We also showed that agerarin is a bioactive component of AHE, responsible for circadian CLOCK-mediated up-regulation of $A Q P 3$. Further work will be needed to examine the therapeutic potential and side effects of agerarin by using animal models of skin wounds to confirm the effects seen in the in vitro experiments presented here. 


\begin{abstract}
Methods
Preparation of A. houstonianum extracts. Young plants of A. houstonianum were purchased from Yangjae flower market (Korea Agro-Fisheries \& Food Trade, Seoul 06749, Republic of Korea) and taxonomically identified by a galenical pharmacist, Dr. Hi Jae Cho (Bio/Molecular Informatics Center, Konkuk University, Seoul, Republic of Korea). They were cultivated in flowerpots for three months in Konkuk University (Seoul, Republic of Korea), as shown in Supplemental Fig. S1. A voucher specimen was deposited at the College of Biological Science and Technology, Konkuk University, Republic of Korea. Air-dried A. houstonianum (1248 g) was immersed in ethanol for 3 days. The filtered solution was dried using a rotary evaporator under a reduced pressure, and the ethanolic extract $(150.9 \mathrm{~g}, 12.09 \%)$ was obtained. This ethanolic extract (120 g) was used for liquid-liquid separation. Three fractions, including ethanol, $\mathrm{n}$-hexane (17.63 g, 14.69\%), chloroform $(6.7 \mathrm{~g}, 5.58 \%)$, and water (93.01 g, $77.51 \%)$ were collected. Each fraction was dried using a freeze-dryer and dissolved in DMSO at $20 \mathrm{mg} / \mathrm{mL}$.
\end{abstract}

Preparative high performance liquid chromatography (prep-HPLC). A prep-HPLC was carried out using an Agilent 1100 instrument with a Luna C18 column $(10 \times 250 \mathrm{~mm}, \varnothing=5 \mu \mathrm{m})$. The mobile phase was a mixture of acetonitrile/methanol/water (v/v 30:50:20), and the flow rate and injection volume were $3.0 \mathrm{~mL} / \mathrm{min}$ and $50 \mu \mathrm{L}$, respectively. The eluents were detected at $230 \mathrm{~nm}$, using a photodiode array detector. The samples were dissolved in isopropanol.

Nuclear magnetic resonance (NMR) spectroscopy and mass spectrometry (MS). For identification of the chemical structure, the following experiments were performed on a Bruker Avance $400 \mathrm{NMR}$ spectrometer (9.4 Tesla; Karlsruhe, Germany): 1D-NMR experiments including ${ }^{1} \mathrm{H}$ NMR, ${ }^{13} \mathrm{C}$ NMR, distortionless enhancement by polarization transfer, and 2D-NMR experiments including COSY, TOCSY, HMQC, and HMBC. The sample was dissolved in $\mathrm{CDCl}_{3}$ and transferred into a 2.5- $\mathrm{mm}$ NMR tube. Detailed experimental procedures were described previously ${ }^{42}$. HR/MS was carried out on a Waters ACQUITY UPLC system (Waters, Milford, MA, USA) with UPLC-Q-Tof-MS as described previously ${ }^{43}$.

Cell culture and reagents. HaCaT cells were obtained from the Cell Lines Service (Eppelheim, Germany) and were maintained in Dulbecco's modified Eagle's medium (DMEM) supplemented with 10\% fetal bovine serum (Hyclone, Logan, UT, USA). Antibodies specific for phospho-ERK1/2 (Thr202/Tyr204), phospho-JNK1/2 (Thr183/Tyr185), and phospho-p38 MAP kinase (Thr180/Tyr182) were purchased from Cell Signaling Technology (Beverly, MA, USA). GAPDH antibody was obtained from Santa Cruz Biotechnology (Santa Cruz, CA, USA). Aquaporin-3 (AQP3) antibody was purchased from BOSTER (Pleasanton, CA, USA). An Alexa Fluor 555-conjugated secondary antibody was obtained from Invitrogen (Carlsbad, CA, USA). The Dual-Glo ${ }^{\circledR}$ Luciferase Assay System for firefly and Renilla luciferase activities was purchased from Promega (Madison, WI, USA). Pierce ${ }^{\mathrm{TM}}$ BCA Protein Assay Reagent was obtained from Thermo Scientific (Rockford, IL, USA). Other chemicals were purchased from Sigma-Aldrich (St. Louis, MO, USA). Plasmid clone of human CLOCK cDNA in the pBluescriptR cloning vector (clone ID:IRAK074P05) was provided by the RIKEN BioResource Center (Tsukuba, Ibaraki, Japan) through the National Bio-Resource Project of the MEXT, Japan.

Reverse transcription-polymerase chain reaction (RT-PCR) and quantitative real-time PCR (qRT-PCR). RT-PCR was performed as described previously ${ }^{44}$. Briefly, reverse transcription was performed using $1 \mu \mathrm{g}$ of total RNA as a template and oligo(dT) primers. The resulting cDNA was subjected to PCR analysis, using gene-specific primers for AQP3 (forward, 5'-CCTTTGGCTTTGCTGTCACTCT-3'; reverse, $5^{\prime}$-CGGGGTTGTTGTAGGGGTCA-3'), CLOCK (forward, $5^{\prime}$-TAGGGTATTTGCCATTTGA-3'; reverse, $5^{\prime}$-GCCAAGTTCTCGTCGTC-3'), and glyceraldehyde-3-phosphate dehydrogenase (GAPDH; forward, $5^{\prime}$-ACCCACTCCTCCACCTTTG-3'; reverse, $5^{\prime}$-CTCTTGTGCTGCTGGG- $3^{\prime}$ ). PCR conditions were as follows: denaturation, $94^{\circ} \mathrm{C}$ for $30 \mathrm{~s}$; annealing, $55^{\circ} \mathrm{C}$ for $30 \mathrm{~s}$; elongation, $72^{\circ} \mathrm{C}$ for $1 \mathrm{~min}$. The amplified products were subjected to electrophoresis on a $1 \%$ agarose gel.

For qRT-PCR, a TaqMan-iQ supermix kit (Bio-Rad) was used according to the manufacturer's instructions. TaqMan ${ }^{\mathrm{TM}}$ fluorogenic probes were designed by Metabion International (Martinsried, Germany). The sequences of primers for qRT-PCR were as follows: AQP3 (forward, 5'-CTTGAGCATCCACTGACT-3'; reverse, 5'-GGGTGAGGGTAGATAGGG-3'; TaqMan probe, 5'-6-FAM-CCCTTCACGATCCACCCTTTCA-BHQ $-3^{\prime}$ ), CLOCK (forward, 5'-CCATCTAGTATGCCACAA-3'; reverse, 5'-TGACCTTGAGAAAATCTTA-3'; TaqMan probe, $5^{\prime}$-FAM-CAGTAACTACATTCACTCAGGACAGGC-BHQ-1-3'), and GAPDH (forward, 5'-TCGACAGTCAGCCGCATCTTC-3'; reverse, 5'-CGCCCAATACGACCACCTCCG-3'; TaqMan probe, 5'-Yakima Yellow TM-CGTCGCCAGCCGAGCCACATCGC-BHQ-1-3'). The relative mRNA expression was analyzed after normalization to GAPDH mRNA using the software program provided by the manufacturer. The data shown represent the mean $\pm \mathrm{SD}(n=3)$.

Immunoblot analysis. Immunoblot analysis was carried out as described previously ${ }^{45}$. Cell lysates $(20 \mu \mathrm{g}$ each) were separated by $10 \%$ SDS-polyacrylamide gel electrophoresis and transferred to nitrocellulose filters. The blots were then incubated with primary antibodies and developed with an enhanced chemiluminescence detection system (GE Healthcare Life Sciences, Piscataway, NJ, USA).

Flow cytometry. Expression of the cell surface AQP3 protein was measured by flow cytometry. AQP3 protein was detected using 1:500 diluted ant-AQP3 antibody (BOSTER). The secondary antibody was 1:500 diluted Rhodamin Red X-conjugated anti-rabbit IgG $(\mathrm{H}+\mathrm{L}$ ) antibody (Jackson ImmunoResearch, West Grove, PA, USA). After washing three times with 5\% bovine serum albumin in PBS, fluorescent intensities were measured on a BD FACS-Calibur (BD Biosciences, San Diego, CA, USA) using the FACSort program. 
Immunofluorescence microscopy. HaCaT keratinocytes were cultured on coverslips and treated with either vehicle (DMSO) or AHE $(20 \mu \mathrm{g} / \mathrm{mL})$ for $30 \mathrm{~min}$, followed by irradiation with ultraviolet B (UVB; $30 \mathrm{~mJ} /$ $\mathrm{m}^{2}$ ) for $24 \mathrm{~h}$. Immunofluorescence analysis was performed as described previously ${ }^{46}$. Primary antibody specific to AQP3 was incubated for $2 \mathrm{~h}$ followed by addition of Alexa-Fluor 555-conjugated secondary antibody (red signal) and further incubated for $1 \mathrm{~h}$. Nuclear DNA (blue signal) was stained with $0.1 \mu \mathrm{g} / \mathrm{mL}$ Hoechst 33258 (Sigma-Aldrich) for $10 \mathrm{~min}$. Staining was examined under an EVOS FL fluorescence microscope (Advanced Microscopy Group; Bothell, WA, USA).

Construction of AQP3 and CLOCK gene promoter reporters. Amplification of the $5^{\prime}$-flanking regions of the $A Q P 3$ and $C L O C K$ genes was performed by PCR, using Dr. Taq DNA Polymerase (Doctor protein, Seoul, Korea). The AQP3 gene was amplified from pGL4-phAQP (RDB07311, Riken DNA bank, Japan), and the CLOCK gene was amplified from the genomic DNA of MDA-MB-231 cells. Nucleotides upstream of the transcription start site were numbered beginning at +1 . An AQP3 promoter fragment spanning positions -1090 to +16 was synthesized using the primers $5^{\prime}$-GGTGAATCCCCATCTCCACT- $3^{\prime}$ (forward) and 5'-CCTTTATAGGAGCGCTGGAG-3' (reverse, +16R). The amplified PCR products were ligated into the HindIII sites of the pGL4.17 vector (Promega), yielding pAQP3-Luc $(-1090 /+16)$. A deletion fragment of AQP3 promoter was synthesized by PCR using the pAQP3-Luc $(-1090 /+16)$ plasmid as a template. Forward primer $5^{\prime}$-CTTGACGTCCCCTCCCTT- $3^{\prime}$ and $+16 \mathrm{R}$ reverse primer were used to generate deletion constructs. The amplified PCR products were ligated into the pGL4-basic vector, yielding pAQP3-Luc $(-198 /+16)$. A CLOCK promoter fragment spanning positions -1000 to +47 was synthesized using the primers $5^{\prime}$-TTTACGGCCAGAAAAGTGCATT-3' (forward) and $5^{\prime}$-TTTACGGCCAGAAAAGTGCATT-3' (reverse). The amplified PCR products were ligated into the KpnI and $B g l \mathrm{II}$ sites of the pGL4.17 vector (Promega), yielding pClock-Luc $(-1000 /+47)$. The resultant constructs were verified by DNA sequencing (Macrogen, Seoul, Korea).

Transient transfection and promoter reporter assays. HaCaT cells seeded onto 12 -well plates were transfected with $A Q P 3$ or $C L O C K$ promoter construct $(0.2 \mu \mathrm{g})$, using Lipofectamine 2000 reagent (Invitrogen Life Technologies, San Diego, CA, USA) according to the manufacturer's instructions. Where indicated, CLOCK expression plasmid (pcDNA3.1/Clock) was also included. Luciferase activities were measured using the Dual-Glo ${ }^{\circledR}$ Luciferase Assay System (Promega), as described previously ${ }^{47}$. Luciferase activity in untreated cells was arbitrarily given a value of 1 (after normalization to the Renilla luciferase signal). Luminescent signal was measured using a Centro LB960 luminometer (Berthold Technologies, Bad Wildbad, Germany).

Silencing of CLOCK expression using small-hairpin RNA (shRNA). HaCaT keratinocytes were infected with shRNA lentiviral particles (MISSION ${ }^{\circledR}$ shRNA; Sigma-Aldrich) expressing scrambled (SHC203V; shCont) or CLOCK shRNA (TRCN0000018978; shClock), following the manufacturer's instructions. Twenty-four hours after infection, puromycin $(2 \mu \mathrm{g} / \mathrm{mL})$ was added for the selection of infected cells. After 2 weeks, cells were collected, and the stable silencing of CLOCK expression was determined by RT-PCR.

Statistical analysis. Statistical analysis was analyzed by one-way analysis of variance (ANOVA) followed by Sidak's multiple comparisons test, using GraphPad Prism version 7.0 software (GraphPad Software Inc., La Jolla, CA, USA). A $P$ value less than 0.05 was considered statistically significant.

Data availability. All data generated or analysed during this study are included in this published article (and its Supplementary Information files).

\section{References}

1. Madison, K. C. Barrier function of the skin: "la raison d'etre" of the epidermis. J Invest Dermatol 121, 231-241 (2003).

2. Sougrat, R. et al. Functional expression of AQP3 in human skin epidermis and reconstructed epidermis. J Invest Dermatol 118, 678-685 (2002)

3. Verkman, A. S., Hara-Chikuma, M. \& Papadopoulos, M. C. Aquaporins-new players in cancer biology. J Mol Med (Berl) 86, 523-529 (2008).

4. Gallant-Behm, C. L. \& Mustoe, T. A. Occlusion regulates epidermal cytokine production and inhibits scar formation. Wound Repair Regen 18, 235-244 (2010).

5. Ghadially, R., Reed, J. T. \& Elias, P. M. Stratum corneum structure and function correlates with phenotype in psoriasis. J Invest Dermatol 107, 558-564 (1996).

6. Sator, P. G., Schmidt, J. B. \& Honigsmann, H. Comparison of epidermal hydration and skin surface lipids in healthy individuals and in patients with atopic dermatitis. J Am Acad Dermatol 48, 352-358 (2003).

7. Segre, J. A. Epidermal barrier formation and recovery in skin disorders. J Clin Invest 116, 1150-1158 (2006).

8. Xu, W. et al. The expression of proinflammatory genes in epidermal keratinocytes is regulated by hydration status. J Invest Dermatol 134, 1044-1055; quiz e1015 (2014).

9. Verkman, A. S. Aquaporins at a glance. J Cell Sci 124, 2107-2112 (2011).

10. Frigeri, A. et al. Localization of MIWC and GLIP water channel homologs in neuromuscular, epithelial and glandular tissues. J Cell Sci 108(Pt 9), 2993-3002 (1995).

11. Ma, T., Hara, M., Sougrat, R., Verbavatz, J. M. \& Verkman, A. S. Impaired stratum corneum hydration in mice lacking epidermal water channel aquaporin-3. J Biol Chem 277, 17147-17153 (2002).

12. Hara, M., Ma, T. \& Verkman, A. S. Selectively reduced glycerol in skin of aquaporin-3-deficient mice may account for impaired skin hydration, elasticity, and barrier recovery. J Biol Chem 277, 46616-46621 (2002).

13. Zeeshan, M., Rizvi, S. M., Khan, M. S. \& Kumar, A. Isolation, partial purification and evaluation of bioactive compounds from leaves of Ageratum houstonianum. EXCLI J 11, 78-88 (2012).

14. Verma, A. et al. Compounds isolated from Ageratum houstonianum inhibit the activity of matrix metalloproteinases (MMP-2 and MMP-9): An oncoinformatics study. Pharmacogn Mag 10, 18-26 (2014). 
15. Wiedenfeld, H. \& Andrade-Cetto, A. Pyrrolizidine alkaloids from Ageratum houstonianum Mill. Phytochemistry 57, 1269-1271 (2001).

16. Kumar, N. Biological potential of a weed Ageratum houstonianum Mill:A review. Indo Am J Pharm Res 4, 2683-2689 (2014).

17. Kurade, N. P., Jaitak, V., Kaul, V. K. \& Sharma, O. P. Chemical composition and antibacterial activity of essential oils of Lantana camara, Ageratum houstonianum and Eupatorium adenophorum. Pharm Biol 48, 539-544 (2010).

18. Pandey, D. K., Chandra, H., Tripathi, N. N. \& Dixit, S. N. Mycotoxicity in leaves of some higher plants with special reference to that of Ageratum houstonianum mill. Mykosen 26, 565-573 (1983).

19. Menut, C., Lamaty, G., Zollo, P. H. A., Kuiate, J. R. \& Bessiere, J. M. Aromatic plants of tropical Central Africa. Part X. Chemical composition of the essential oils of Ageratum houstonianum Mill. and Ageratum conyzoides L. from Cameroon. Flavour Fragrance J 8, 1-4 (1993).

20. Singh, B. \& Singh., J. Ethnobotanical uses of some plants from central Haryana, India. Phytodiversity 1, 7-24 (2014).

21. Cao, C. et al. EGFR-mediated expression of aquaporin-3 is involved in human skin fibroblast migration. Biochem J 400, 225-234 (2006).

22. Cao, C. et al. All-trans retinoic acid attenuates ultraviolet radiation-induced down-regulation of aquaporin-3 and water permeability in human keratinocytes. J Cell Physiol 215, 506-516 (2008).

23. Yoon, H. et al. 1H and 13C NMR spectral assignments of novel chromenylchalcones. Magn Reson Chem 50, 759-764 (2012).

24. Kasturi, T. R., Abraham, E. M. \& Brown, P. Structure of a dimer of ageratochromene. J Chem Soc, Perkin Trans 1, 2468-2470 (1973).

25. Pratt, G. E. \& Bowers, W. S. Precocene II inhibits juvenile hormone biosynthesis by cockroach corpora allata in vitro. Nature 265, 548-550 (1977).

26. Matsunaga, N. et al. 24-hour rhythm of aquaporin-3 function in the epidermis is regulated by molecular clocks. J Invest Dermatol 134, 1636-1644 (2014)

27. Verkman, A. S. Aquaporins: translating bench research to human disease. J Exp Biol 212, 1707-1715 (2009).

28. Yosipovitch, G. et al. Time-dependent variations of the skin barrier function in humans: transepidermal water loss, stratum corneum hydration, skin surface $\mathrm{pH}$, and skin temperature. J Invest Dermatol 110, 20-23 (1998).

29. Reppert, S. M. \& Weaver, D. R. Coordination of circadian timing in mammals. Nature 418, 935-941 (2002).

30. Gery, S. \& Koeffler, H. P. Circadian rhythms and cancer. Cell Cycle 9, 1097-1103 (2010).

31. Huang, W., Ramsey, K. M., Marcheva, B. \& Bass, J. Circadian rhythms, sleep, and metabolism. J Clin Invest 121, 2133-2141 (2011).

32. He, B. et al. The Small Molecule Nobiletin Targets the Molecular Oscillator to Enhance Circadian Rhythms and Protect against Metabolic Syndrome. Cell Metab 23, 610-621 (2016).

33. Zhang, E. E. \& Kay, S. A. Clocks not winding down: unravelling circadian networks. Nat Rev Mol Cell Biol 11, 764-776 (2010).

34. Hastings, M. H., Reddy, A. B. \& Maywood, E. S. A clockwork web: circadian timing in brain and periphery, in health and disease. Nat Rev Neurosci 4, 649-661 (2003).

35. Dunlap, J. C. Molecular bases for circadian clocks. Cell 96, 271-290 (1999).

36. Zanello, S. B., Jackson, D. M. \& Holick, M. F. Expression of the circadian clock genes clock and period1 in human skin. J Invest Dermatol 115, 757-760 (2000).

37. Sporl, F. et al. A circadian clock in HaCaT keratinocytes. J Invest Dermatol 131, 338-348 (2011).

38. Goldsmith, C. S. \& Bell-Pedersen, D. Diverse roles for MAPK signaling in circadian clocks. Adv Genet 84, 1-39 (2013).

39. Hayashi, Y., Sanada, K., Hirota, T., Shimizu, F. \& Fukada, Y. p38 mitogen-activated protein kinase regulates oscillation of chick pineal circadian clock. J Biol Chem 278, 25166-25171 (2003).

40. Vrailas-Mortimer, A. D. et al. p38 MAP kinase regulates circadian rhythms in Drosophila. J Biol Rhythms 29, 411-426 (2014).

41. Ryu, H. M. et al. Aquaporin 3 expression is up-regulated by TGF-betal in rat peritoneal mesothelial cells and plays a role in wound healing. Am J Pathol 181, 2047-2057 (2012).

42. Yong, Y. et al. 1H and 13C NMR spectral assignments of 2'-hydroxychalcones. Magn Reson Chem 51, 364-370 (2013).

43. Ahn, S. et al. (1) H and (13) C NMR spectral assignments of novel flavonoids bearing benzothiazepine. Magn Reson Chem 54, 382-390 (2016)

44. Shin, S. Y., Kim, C. G., Jung, Y. J., Lim, Y. \& Lee, Y. H. The UPR inducer DPP23 inhibits the metastatic potential of MDA-MB-231 human breast cancer cells by targeting the Akt-IKK-NF-kappaB-MMP-9 axis. Sci Rep 6, 34134 (2016).

45. Lee da, H. et al. A synthetic chalcone, $2^{\prime}$-hydroxy-2,3,5'-trimethoxychalcone triggers unfolded protein response-mediated apoptosis in breast cancer cells. Cancer Lett 372, 1-9 (2016).

46. Shin, S. Y. et al. Inhibition of PDGF-induced migration and TNF-alpha-induced ICAM-1 expression by maltotetraose from bamboo stem extract (BSE) in mouse vascular smooth muscle cells. Mol Nutr Food Res 60, 2086-2097 (2016).

47. Lee, M. S. et al. 2-Hydroxy-3,4-naphthochalcone (2H-NC) inhibits TNFalpha-induced tumor invasion through the downregulation of NF-kappaB-mediated MMP-9 gene expression. Bioorg Med Chem Lett 25, 128-132 (2015).

\section{Acknowledgements}

This study was supported by the Basic Science Research Program through the National Research Foundation of Korea (NRF) funded by the Ministry of Science, ICT and Future Planning (grant no. 2016R1A2B4008570) and by the Cooperative Research Program for Agriculture Science \& Technology Development (project no. PJ011884022016; Rural Development Administration), Republic of Korea. This paper was supported by the KU Research Professor Program of Konkuk University.

\section{Author Contributions}

S.Y.S., Y.L., and Y.H.L. participated in the study design. B.S.K., J.-S.C., and J.-B.K. isolated and fractionated plant extracts. J.-B.K. and Y.L. carried out HPLC and NMR experiments. S.Y.S., H.-N.G., and D.H.L. performed molecular and cellular experiments. S.Y.S., Y.L., and Y.H.L. wrote the manuscript. All authors reviewed the manuscript.

\section{Additional Information}

Supplementary information accompanies this paper at doi:10.1038/s41598-017-11642-X

Competing Interests: The authors declare that they have no competing interests.

Publisher's note: Springer Nature remains neutral with regard to jurisdictional claims in published maps and institutional affiliations. 
(i) Open Access This article is licensed under a Creative Commons Attribution 4.0 International License, which permits use, sharing, adaptation, distribution and reproduction in any medium or format, as long as you give appropriate credit to the original author(s) and the source, provide a link to the Creative Commons license, and indicate if changes were made. The images or other third party material in this article are included in the article's Creative Commons license, unless indicated otherwise in a credit line to the material. If material is not included in the article's Creative Commons license and your intended use is not permitted by statutory regulation or exceeds the permitted use, you will need to obtain permission directly from the copyright holder. To view a copy of this license, visit http://creativecommons.org/licenses/by/4.0/.

(C) The Author(s) 2017 